\title{
Indução Anestésica após o Tratamento do Choque Hemorrágico: Estudo Experimental Comparando a Cetamina e o Etomidato
}

\author{
Anesthetic Induction after Treated Hemorrhagic Shock: \\ Experimental Study Comparing Ketamine and Etomidate
}

\author{
Adilson O. Fraga', Luiz Marcelo Sá Malbouisson, TSA², Ricardo Prist ${ }^{3}$, Maurício Rocha e Silva ${ }^{4}$, \\ José Otávio Costa Auler Júnior, TSA
}

\section{RESUMO}

Fraga AO, Malbouisson LMS, Prist R, Silva MR, Auler Jr JOC Indução Anestésica após o Tratamento do Choque Hemorrágico: Estudo Experimental Comparando a Cetamina e o Etomidato.

JUSTIFICATIVA E OBJETIVOS: O sangramento que leva ao choque hemorrágico geralmente necessita tratamento cirúrgico sob anestesia geral. Por sua vez, os anestésicos podem comprometer ainda mais as condições hemodinâmicas. O objetivo deste estudo foi o de comparar os efeitos hemodinâmicos da cetamina e do etomidato durante a indução anestésica em cães submetidos a um modelo experimental de choque hemorrágico e reanimação.

MÉTODO: Trinta e dois cães mestiços foram submetidos ao choque hemorrágico pressão-controlado, reanimação e indução anestésica. Após atingir a pressão alvo de $40 \mathrm{mmHg}$ eles foram divididos aleatoriamente em dois grupos de acordo com a solução

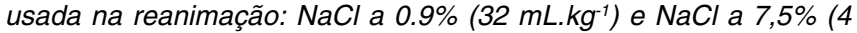
$\left.\mathrm{mL} . \mathrm{kg}^{-1}\right)$. Após a infusão de volume, esses grupos foram divididos novamente de acordo com o anestésico utilizado: GI) $\mathrm{NaCl}$ a $0.9 \%$ e cetamina; GII) NaCl a $7.5 \%$ e cetamina; GIII) NaCl a $0.9 \%$ e etomidato; e GIV) $\mathrm{NaCl}$ a $7.5 \%$ e etomidato. Medições hemodinâmicas foram obtidas em cinco momentos: (MO) inicial; (M1) após o desenvolvimento do choque hemorrágico; (M2) após a administração de fluidos; (M3) 5 minutos após a indução anestésica; (M4) 15 minutos após a indução anestésica. Foi feita a análise estatística usando o teste $t$ de Student e two-way ANOVA. Foram considerados significativos valores de $p$ menores do que 0,05.

RESULTADOS: Após a instalação do choque, ambas as soluções restabeleceram os padrões hemodinâmicos aos valores iniciais. Independente do anestésico ou da solução utilizados, após a indução

${ }^{*}$ Recebido (Received from) da Disciplina de Anestesiologia da Faculdade de Medicina da Universidade de São Paulo (FMUSP), São Paulo, SP. Tese de Mestrado apresentada na FMUSP.

1. Médico Assistente do Serviço de Anestesiologia do InCor da FMUSP.

2. Doutor em Ciências pela USP. Especialista em Terapia Intensiva - AMIB; Médico Assistente do Serviço de Anestesiologia e Terapia Intensiva Cirúrgica do InCor - HCFMUSP.

3. Doutor em Medicina Veterinária - USP - Médico da Divisão de Experimentação HCFMUSP.

4. Professor Titular Dept ${ }^{\circ}$ de Cardiopneumologia da FMUSP; Diretor da Divisão Experimental do InCor HCFMUSP.

5. Professor Titular da Disciplina de Anestesiologia da FMUSP; Especialista em Terapia Intensiva - AMIB. Diretor do Serviço de Anestesiologia e Terapia Intensiva Cirúrgica do InCor - HCFMUSP.

Apresentado (Submitted) em 24 de outubro de 2005 Aceito (Accepted) para publicação em 28 de abril de 2006

Endereço para correspondência (Correspondence to):

Dr. José Otávio Costa Auler Júnior

Av. Enéas de Carvalho Aguiar, 44

05403-000 São Paulo, SP

Email: auler@incor.usp.br

(c) Sociedade Brasileira de Anestesiologia, 2006. anestésica a pressão arterial média permaneceu inalterada em todos os grupos. A pressão venosa central, freqüência cardíaca, pressão capilar pulmonar e o índice de resistência pulmonar vascular aumentaram significativamente após a administração de cetamina. $O$ índice cardíaco, o índice de resistência vascular sistêmica e o transporte de oxigênio permaneceram estáveis em todos os grupos. CONCLUSÕES: $O$ etomidato ou a cetamina foram capazes de manter a estabilidade hemodinâmica nos cães que sofreram choque hemorrágico intenso e que foram tratados com $\mathrm{NaCl}$ a $0,9 \%$ ou $\mathrm{NaCl}$ a $7,5 \%$.

Unitermos: ANIMAIS: cães; COMPLICAÇÕES: choque hemorrágico; DROGAS: cetamina, etomidato; TERAPÊUTICA: reanimação volêmica; VOLEMIA: solução fisiológica a $0,9 \%, \mathrm{NaCl} 7,5 \%$.

\section{SUMMARY}

Fraga AO, Malbouisson LMS, Prist R, Silva MR, Auler Jr JOC Anesthetic Induction after Treated Hemorrhagic Shock: Experimental Study Comparing Ketamine and Etomidate.

BACKGROUND AND OBJECTIVES: Bleeding causing hemorrhagic shock usually requires surgical treatment under general anesthesia. Anesthetic drugs may further compromise hemodynamics. The objective was to compare the hemodynamic effects of ketamine and etomidate during anesthetic induction in dogs submitted to an experimental model of hemorrhagic shock and resuscitation.

METHODS: Thirty-two mongrel dogs were submitted to a pressurecontrolled hemorrhagic shock, resuscitation and anesthetic induction model. After achieving the target pressure of $40 \mathrm{mmHg}$, they were randomly assigned in two groups according to the resuscitation fluid to be used: $\mathrm{NaCl} 0.9 \%\left(32 \mathrm{~mL} . \mathrm{kg}^{-1}\right)$ and $\mathrm{NaCl} 7.5 \%\left(4 \mathrm{~mL} . \mathrm{kg}^{-1}\right)$. After volume infusion, these groups were reassigned according to anesthetic drug used: $\mathrm{Gl}$ ) $\mathrm{NaCl} 0.9 \%$ and ketamine; GII) $\mathrm{NaCl} 7.5 \%$ and ketamine; GIII) $\mathrm{NaCl} 0.9 \%$ and etomidate; and GIV) $\mathrm{NaCl} 7.5 \%$ and etomidate. Hemodynamic measurements were obtained at five moments: (M0) baseline; (M1) after bleeding to shock; (M2) after volume expansion; (M3) 5 minutes after anesthetic induction; (M4) 15 minutes after anesthetic induction. Statistical analysis was performed using Student $t$ test and two way ANOVA. Value of $p$ lower than 0.05, was considered significant.

RESULTS: After shock, both solutions restored hemodynamics to baseline values. Independently of anesthetic agent or expansion solution used, mean arterial pressure remained unaltered for all groups after induction. Central venous pressure, heart rate, pulmonary capillary wedge pressure and pulmonary vascular resistance index increased significantly after ketamine infusion. Cardiac index, systemic vascular resistance index and oxygen transport variables remained stable in all groups.

CONCLUSIONS: Etomidate or ketamine were able to maintain hemodynamic stability in dogs undergoing severe hemorrhagic shock treated with $\mathrm{NaCl} 0.9 \%$ or $\mathrm{NaCl} 7.5 \%$.

Key Words: ANIMALS: dogs; COMPLICATIONS: hemorrhagic shock; DRUGS: etomidate, ketamine; THERAPEUTIC: volemic resuscitation; VOLEMIA: $0.9 \%$ saline solution, $7.5 \% \mathrm{NaCl}$. 


\section{INTRODUÇÃO}

$\mathrm{O}^{\circ}$ choque hemorrágico causado pelo trauma é uma importante causa de internação hospitalar de emergência. Em geral, é necessária intervenção cirúrgica para controlar o sangramento e a indução anestésica pode acentuar a instabilidade hemodinâmica devido à vasodilatação e à depressão do miocárdio. Nesses pacientes, a administração de grandes quantidades de volume usando soluções isotônicas ou hipertônicas tem sido utilizada para a reanimação volêmica inicial na emergência antes da intervenção cirúrgica ${ }^{1,2}$. Estudos experimentais e clínicos mostraram que a solução hipertônica de cloreto de sódio é eficaz na restauração da estabilidade hemodinâmica após o choque hemorrágico ${ }^{3-5}$, mas a solução isotônica de cloreto de sódio continua a ser a solução usada com mais freqüência na reanimação inicial do choque hemorrágico ${ }^{1}$. Ainda existe uma controvérsia sobre qual o melhor medicamento para a indução anestésica que interfira o mínimo possível no estado hemodinâmico dos pacientes hipovolêmicos após um trauma ${ }^{6,7}$. Em 1990, Haskins e col. estudaram os efeitos da cetamina em um modelo de hipovolemia em cães, observando que ela mantinha uma função cardiovascular adequada, preservava o transporte de oxigênio e causava apenas depressão respiratória temporária ${ }^{8}$. Com base nessa informação, a cetamina foi considerada uma opção viável para a indução anestésica de pacientes hipovolêmicos em uma emergência. $O$ etomidato, outro anestésico, foi considerado seguro para ser administrado em pacientes graves, pois os reflexos do sistema nervoso autônomo são preservados, assim como a contratilidade do miocárdio ${ }^{6}$. Apesar do uso generalizado desses dois anestésicos em casos de instabilidade hemodinâmica, poucos estudos compararam esses medicamentos em modelos experimentais de choque hemorrágico e sua interação com a solução fisiológica normal e a hipertônica usadas no tratamento do choque hemorrágico ${ }^{8-10}$.

O objetivo desse estudo foi o de comparar os efeitos hemodinâmicos da cetamina com os do etomidato em um modelo de choque hemorrágico em cachorros após a reanimação com solução fisiológica normal ou hipertônica ( $\mathrm{NaCl}$ a $7,5 \%)$.

\section{MÉTODO}

Este estudo foi aprovado pelo Comitê de Ética do hospital, seguindo as regras internacionais para pesquisa com animais. Foram usados 32 cães mestiços pesando de 15 a 20 $\mathrm{kg}$. Os cães permaneceram em jejum por 12 horas antes do início do estudo, mas podiam beber água à vontade.

Para evitar uma possível influência de outro anestésicos, os animais foram sedados com tiopental $\left(15 \mathrm{mg}^{\mathrm{kg}} \mathrm{kg}^{-1}\right)$ e instituída a ventilação mecânica 24 horas antes do experimento. Um cateter arterial pulmonar 7-French (Baxter Healthcare Corp., Irvine, CA, EUA) foi introduzido na veia jugular interna direita e avançado até a artéria pulmonar com o auxílio de um fluoroscópio. Cateteres de polietileno de pequeno calibre (P260) contendo heparina $10 \mathrm{UI} . \mathrm{mL}^{-1}$ foram introduzidos, por meio de dissecção, nas artérias e nas veias femorais. Os cateteres arteriais foram usados para medir a pressão arterial sistêmica, retirar sangue e colher amostras de sangue arterial, assim como para a administração de medicamentos e de soluções. Todos os cateteres foram presos com firmeza, passando por um túnel subcutâneo e exteriorizados na região dorsal de cada animal para serem usados posteriormente. Depois que todos os acessos vasculares foram estabelecidos, foi administrada heparina 50 Ul. $\mathrm{kg}^{-1}$ pela via venosa central, os cães foram extubados e tiveram um período de recuperação de 24 horas durante o qual o consumo de água foi liberado. Após esse período de repouso os cães foram medicados com morfina $(0,4$ mg. $\left.\mathrm{kg}^{-1}\right)$ uma hora antes de serem transportados para a sala onde o estudo seria conduzido.

Foram determinados os níveis de oxigênio arterial e venoso $\left(\mathrm{PaO}_{2}, \mathrm{PvO}_{2}\right)$, a tensão de gás carbônico arterial e venosa $\left(\mathrm{PaCO}_{2}, \mathrm{PvCO}_{2}\right)$ e o sódio plasmático $\left(\mathrm{Na}^{+}\right)$, assim como $\circ \mathrm{pH}$ arterial e venoso usando-se um analisador de gasometria sangüínea (Gas Analyzer ABL - Radiometer, Copenhagen). Os resultados foram corrigidos para a temperatura corporal do animal, sendo calculados a concentração de bicarbonato de sódio e o excesso de base para cada animal. Também foram determinadas as concentrações de hemoglobina $(\mathrm{Hb})$. A pressão arterial média (PAM), a pressão arterial pulmonar média (PAPM), a pressão capilar pulmonar (PCP) e a pressão venosa central (PVC) foram medidas, usando-se transdutores de pressão, pelo Acknowledge MP 100 (Biopac Systems Inc., Goleta, CA). O débito cardíaco $(\mathrm{DC})$ foi determinado com a utilização da técnica-padrão de termodiluição. Foram feitas cinco medições e o valor médio foi dividido pela área da superfície corporal para obtenção do índice cardíaco (IC). As resistências vasculares periférica e pulmonar, o conteúdo de oxigênio no sangue arterial e venoso $\left(\mathrm{CaO}_{2}\right.$ a $\left.\mathrm{CvO}_{2}\right)$, a mistura venosa, o consumo de oxigênio $\left(\mathrm{VO}_{2}\right)$, a oferta de oxigênio e a taxa de utilização de oxigênio foram calculados a partir dos resultados obtidos por meio das equações convencionais.

O modelo de choque hemorrágico utilizado foi descrito por Prist e col. ${ }^{11}$. Nesse modelo o animal foi submetido a sangramento contínuo durante o procedimento e uma pequena amostra de sangue foi colhida a cada minuto. A pressão durante os últimos 30 segundos (30s) do minuto anterior a cada sangramento foi usada para estabelecer o volume de sangue que deveria ser removido durante os primeiros 30 s do próximo minuto. Assim, o volume da amostra de sangue corresponde à PAM prévia. O modelo descrito tem como base uma situação-padrão, na qual a remoção de um volume inicial de sangue de $25 \mathrm{~mL}$. $\mathrm{min}^{-1}$ de cães pesando $17,5 \mathrm{~kg}$ com uma PAM de $100 \mathrm{mmHg}$ corresponde a uma perda sangüínea de $100 \mathrm{~mL} \cdot \mathrm{min}^{-1} \mathrm{em}$ um adulto de $70 \mathrm{~kg}$ com a mesma PAM. Foram levados em consideração 
os pesos dos diversos animais ou as variações da PAM ao determinar a velocidade de sangramento de acordo com as seguintes condições:

1. A PAM foi determinada durante os 5 minutos que precediam o início da hemorragia;

2. Um volume inicial de sangramento $(\mathrm{VbO})$ foi adaptado ao peso corporal;

Já que o alvo do choque é atingir uma pressão arterial média de $40 \mathrm{mmHg}$ durante o sangramento, espera-se que ocorra uma variação individual no volume sangüíneo total eliminado para que seja mantida a mesma PAM.

Os animais foram estudados com relação a diversos parâmetros hemodinâmicos avaliados nos cinco momentos do experimento, de M0 - inicial para M4.

(M0) Inicial;

(M1) Após o sangramento (choque hemorrágico);

(M2) Após a reanimação volêmica (soluções de cloreto de sódio isotônica e hipertônica);

(M3) 5 minutos após a administração do anestésico (etomidato ou cetamina) e intubação traqueal;

(M4)15 minutos após a administração do anestésico.

A figura 1 mostra o procedimento experimental. Após a obtenção dos valores hemodinâmicos iniciais (M0), foi induzido choque hemorrágico nos animais de acordo com o procedimento descrito na seção do protocolo hemorrágico. Após atingir a meta de pressão arterial média de $40 \mathrm{mmHg}$, 30 minutos após o início da experiência (M1), foram obtidas novas medições hemodinâmicas e amostras sangüíneas. Nesse momento os animais foram divididos em dois gru- pos, de acordo com a solução venosa utilizada: 16 cães receberam $\mathrm{NaCl}$ a $0,9 \%\left(32 \mathrm{~mL} . \mathrm{kg}^{-1}\right)$ e 16 receberam $\mathrm{NaCl}$ a $7,5 \%$ (4 mL. $\left.\mathrm{kg}^{-1}\right)$ durante dez minutos. Os volumes das soluções isotônica e hipertônica foram escolhidos de forma a fornecer a mesma quantidade de $\mathrm{NaCl}$ aos dois grupos. Após a expansão volêmica (M2), foram obtidas novas medições hemodinâmicas e amostras de sangue arterial e venoso foram colhidas. No próximo passo, cada grupo foi dividido em dois subgrupos que receberam etomidato $\left(1 \mathrm{mg}^{\mathrm{kg}}{ }^{-1}, \mathrm{n}\right.$ $=8$ ) ou cetamina ( $4 \mathrm{mg} \cdot \mathrm{kg}^{-1}, \mathrm{n}=8$ ) para indução anestésica, seguida da intubação traqueal. As medições de M3 e M4 foram obtidas 5 e 15 minutos após a administração do anestésico, respectivamente. Após M2 os quatro grupos de animais foram denominados $\mathrm{Gl}$ ) $\mathrm{NaCl}$ a $0,9 \%$ e cetamina; GII) $\mathrm{NaCl}$ a $7,5 \%$ e cetamina; GIII) $\mathrm{NaCl}$ a $0,9 \%$ e etomidato; e GIV) $\mathrm{NaCl}$ a $7,5 \%$ e etomidato.

As análises estatísticas foram feitas usando-se o pacote estatístico SPSS 10 (SPSS Inc., Cary, CA). One-way ANOVA foi utilizada para comparar peso, área da superfície corporal e perda total de sangue entre os quatro grupos. Two-way ANOVA foi usada para as medidas de repetição seguida do Student-Newmann post-host teste, quando indicado, para analisar os parâmetros hemodinâmicos. Um valor de $p<$ 0,05 foi considerado estatisticamente significativo. Todas as informações são apresentadas como a média \pm DP.

Durante o período inicial (M0) e M1 todos os animais foram submetidos aos mesmos procedimentos e os mesmos dados foram colhidos. Para análise estatística eles foram considerados como um único grupo. Durante M2, para a

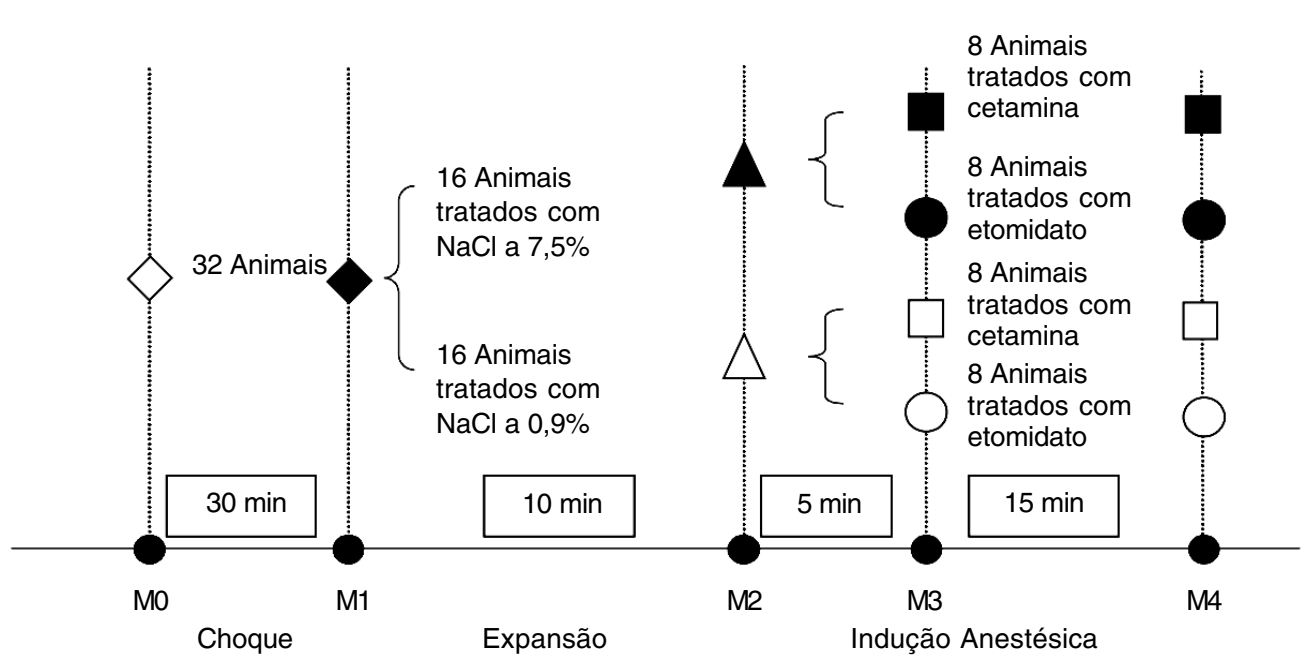

Figura 1 - Modelo Experimental.

M0: inicial; M1: 30 minutos após o sangramento; M2: 10 minutos após a reposição volêmica; M3: 5 minutos após a indução anestésica e a intubação traqueal; M4: 15 minutos após a indução anestésica e a intubação traqueal. Losangos abertos (todos os animais - inicial), losangos fechados (todos os animais - após o choque), triângulos abertos (animais tratados com $\mathrm{NaCl}$ a 0,9\%), triângulos fechados (animais tratados com NaCl a 7,5\%), quadrados abertos (GI - animais tratados com NaCl a 0,9\% e cetamina), quadrados fechados (Gll - animais tratados com $\mathrm{NaCl}$ a 7,5\% e cetamina), círculos abertos (GIII - animais tratados com NaCl a 0,9\% e etomidato) e círculos fechados (GIV - animais tratados com $\mathrm{NaCl}$ a $7,5 \%$ e etomidato). 
análise estatística, os animais foram divididos em dois grupos, de acordo com a solução venosa administrada: 16 animais receberam $\mathrm{NaCl}$ a $0,9 \%$ e 16 animais receberam $\mathrm{NaCl}$ a 7,5\%. Após M2 cada grupo de 16 animais foi subdividido em dois grupos $(n=8)$ de acordo com o anestésico recebido, etomidato ou cetamina. Durante M3 e M4 os grupos, previamente definidos como Gl) $\mathrm{NaCl}$ a $0,9 \%$ e cetamina; GII) $\mathrm{NaCl}$ a $7,5 \%$ e cetamina; GIII) $\mathrm{NaCl}$ a $0,9 \%$ e etomidato; e GIV) $\mathrm{NaCl}$ a 7,5\% e etomidato, foram submetidos a análises estatísticas.

\section{RESULTADOS}

A tabela I apresenta um resumo das características dos animais, incluindo peso, área da superfície corporal e o volume de sangue retirado. Não houve diferença estatística significativa entre os grupos quanto aos valores da área da superfície corporal e o volume de sangue perdido.

As figuras 2, 3 e 4 mostram que foram observados sinais hemodinâmicos de acentuada perda sangüínea. Comparado com a fase inicial (MO) houve redução significativa na PVC, PAM, IC, PCP e PAPM $(p<0,001)$ e aumentos significativos na IRVP e IRVS $(p<0,001)$, enquanto a FC permaneceu inalterada. Houve redução significativa na oferta de oxigênio $\left(\mathrm{IDO}_{2}\right)$ e no consumo de oxigênio $\left(\mathrm{VO}_{2}\right)(\mathrm{p}<0,001)$ e um aumento importante na extração de oxigênio $\left(E_{2}\right)(p$ $<0,001)$.

Figura 2 - Pressão Arterial Média (PAM), Pressão Arterial Pulmonar Média (PAPM), Pressão Venosa Central (PVC) e Pressão Capilar Pulmonar (PCP).

Tratamento dado em M2. Losangos abertos (todos os animais inicial), losangos fechados (todos os animais - após o choque), triângulos abertos (animais tratados com $\mathrm{NaCl}$ a $0,9 \%$ ), triângulos fechados (animais tratados com $\mathrm{NaCl}$ a $7,5 \%$ ), quadrados abertos (Gl - animais tratados com $\mathrm{NaCl}$ a $0,9 \%$ e cetamina), quadrados fechados (GII - animais tratados com $\mathrm{NaCl}$ a $7,5 \%$ e cetamina), círculos abertos (GIII - animais tratados com $\mathrm{NaCl}$ a $0,9 \%$ e etomidato) e círculos fechados (GIV - animais tratados com $\mathrm{NaCl}$ a $7,5 \%$ e etomidato).

$\left(^{*}\right)$ diferença significativa $(p<0,05)$ com relação à inicial.

$(+)$ diferença significativa entre os grupos tratados com cetamina e etomidato $(p<0,05)$.

$(\Delta)$ diferenças significativas entre Gll e os outros grupos após a indução anestésica $(p<0,05)$.

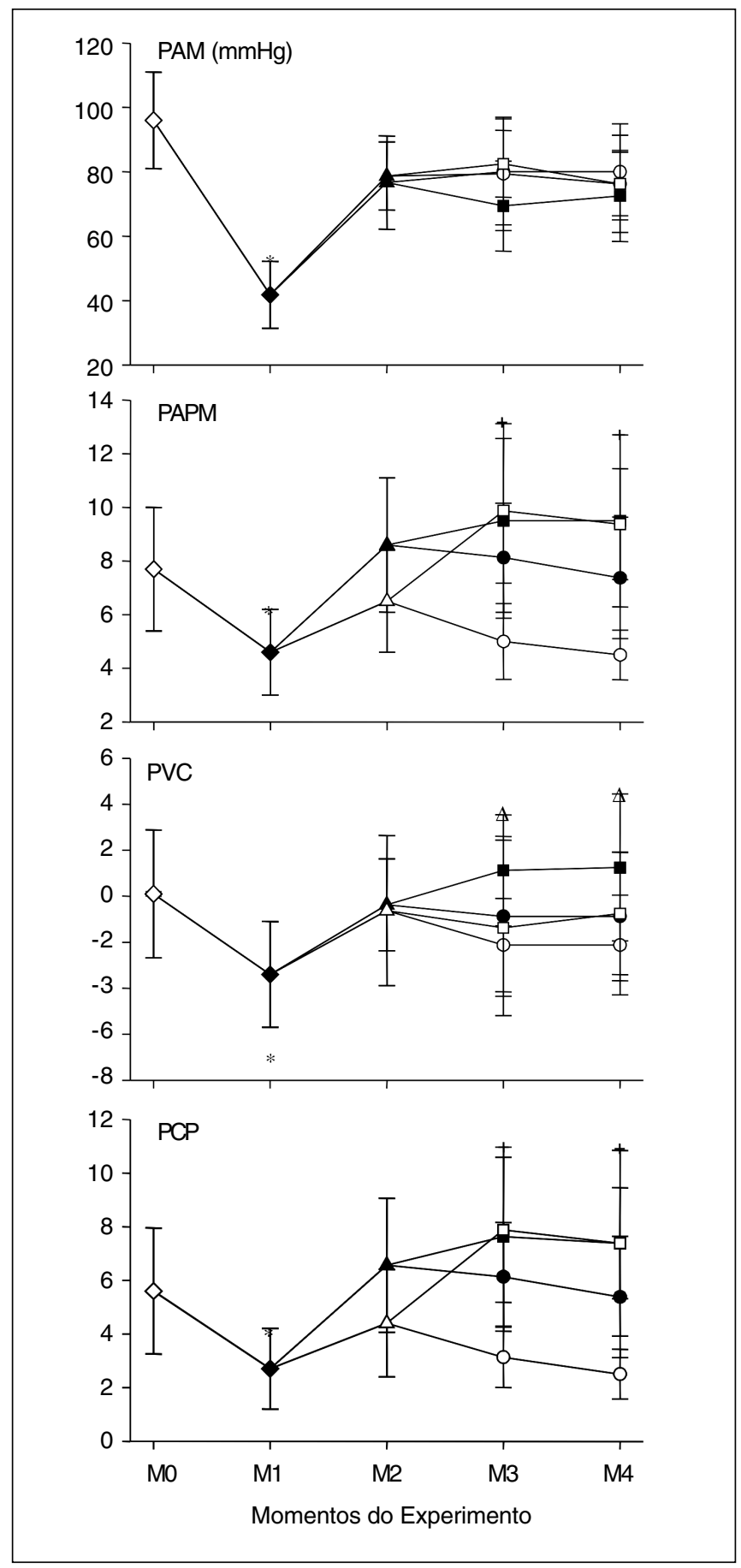

Tabela I - Características dos Animais (Média \pm DP)

\begin{tabular}{lcccc}
\hline & Grupo I $(\mathrm{n}=8)$ & Grupo II $(\mathrm{n}=8)$ & Grupo III $(\mathrm{n}=8)$ & Grupo IV $(\mathrm{n}=8)$ \\
\hline Peso $(\mathrm{kg})$ & $17,36 \pm 0,37$ & $17,46 \pm 0,50$ & $17,31 \pm 0,49$ & $17,79 \pm 0,54$ \\
Área da superfície corporal $\left(\mathrm{kg} \cdot \mathrm{m}^{-2}\right)$ & $0,82 \pm 0,01$ & $0,82 \pm 0,16$ & $0,82 \pm 0,15$ & $0,83 \pm 0,17$ \\
Volume total de sangue perdido $\left(\mathrm{mL} . \mathrm{kg}^{-1}\right)$ & $28,84 \pm 1,47$ & $28,92 \pm 1,75$ & $30,44 \pm 2,0$ & $30,01 \pm 1,81$ \\
\hline
\end{tabular}

Grupo I: solução fisiológica ( $\mathrm{NaCl}$ a 0,9\%) e cetamina; Grupo II: solução hipertônica ( $\mathrm{NaCl}$ a 7,5\%) e cetamina: Grupo III: $\mathrm{NaCl}$ a $0,9 \%$ e etomidato; Grupo IV: $\mathrm{NaCl}$ a $7,5 \%$ e etomidato 


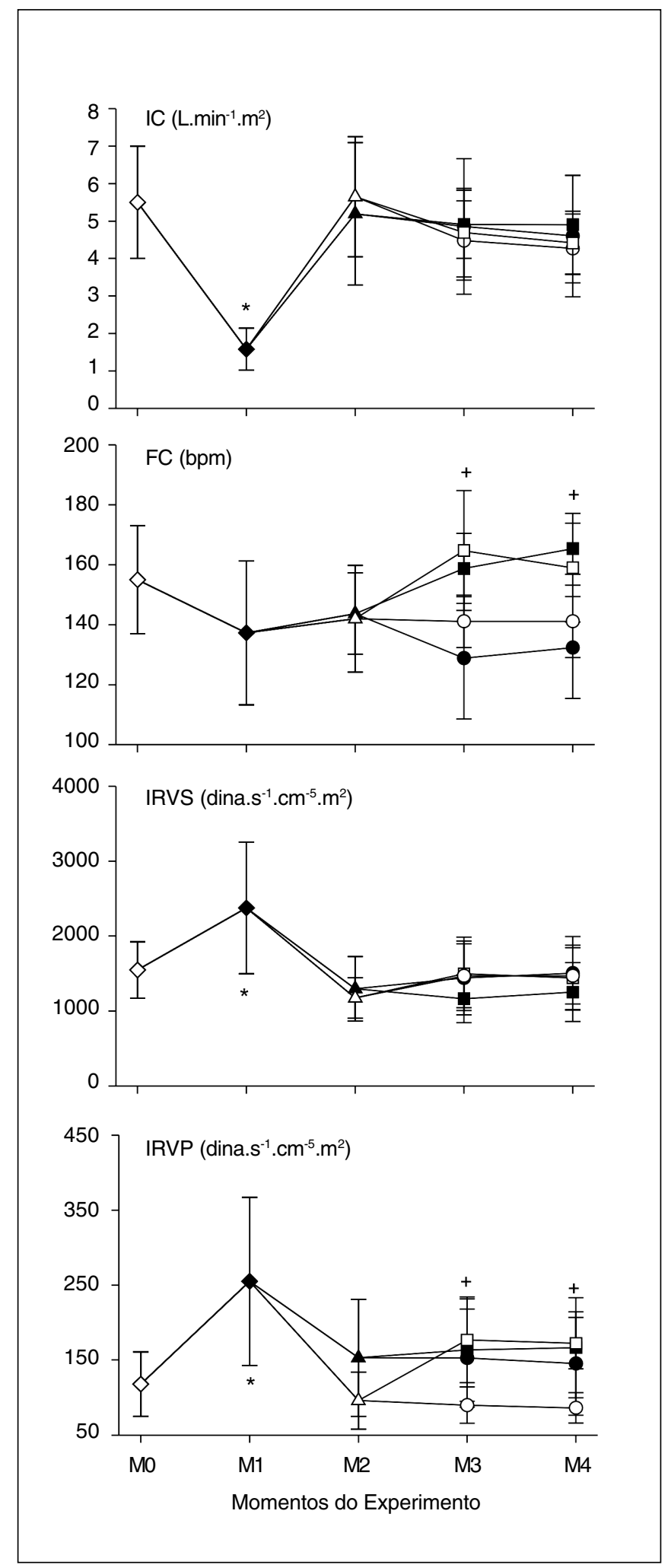

Figura 3 - Índice Cardíaco (IC), Freqüência Cardíaca (FC), Índice de Resistência Vascular Sistêmica (IRVS) e Índice de Resistência Vascular Pulmonar (IRVP)

$\left(^{*}\right)$ diferença significativa $(p<0,05)$ com relação à inicial.

(+) diferença significativa entre os grupos tratados com cetamina e etomidato $(p<0,05)$.

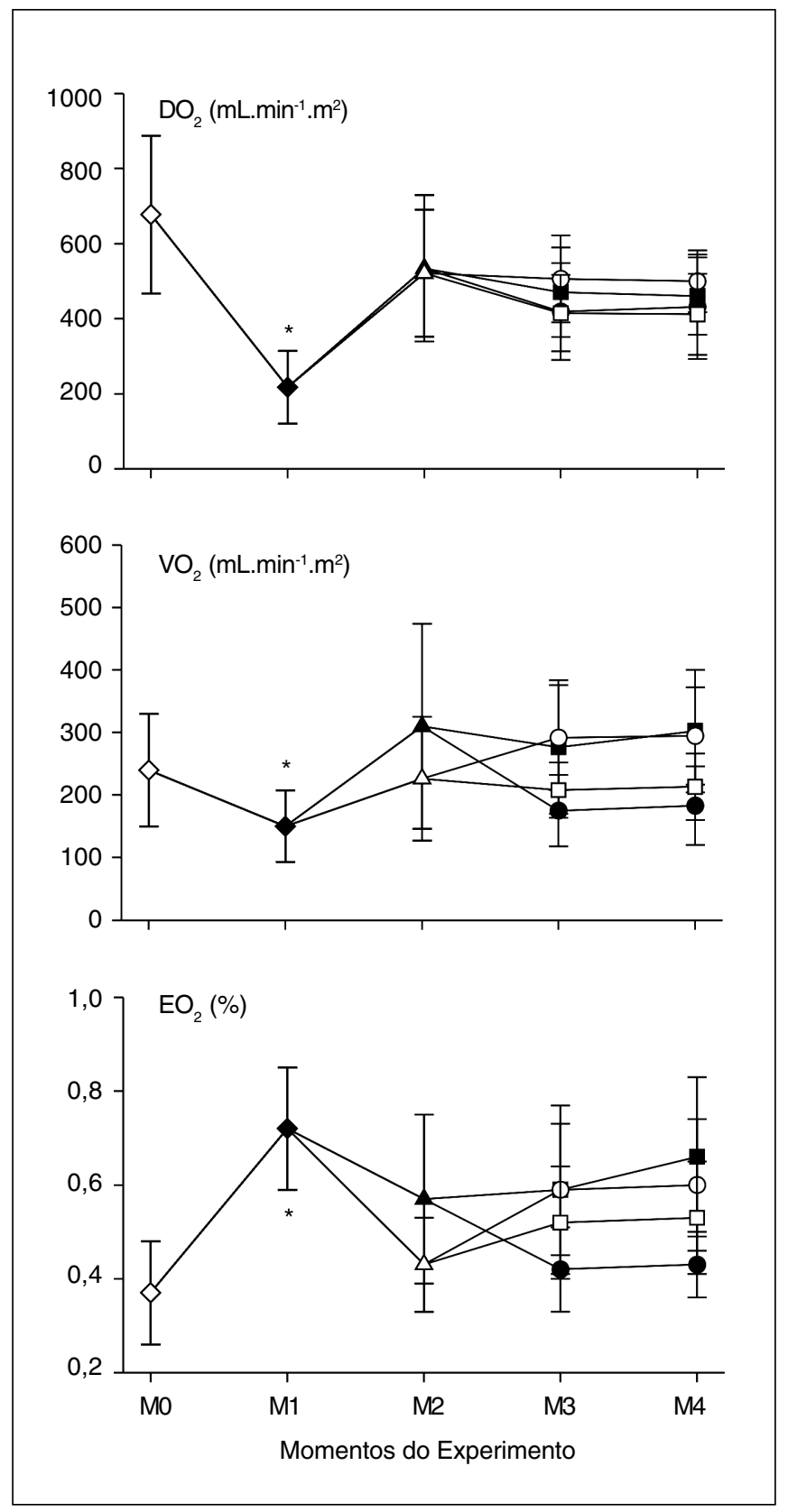

Figura 4 - Índice de Transporte de Oxigênio $\left(\mathrm{DO}_{2}\right)$, Índice de Consumo de Oxigênio $\left(\mathrm{VO}_{2}\right)$, Extração de Oxigênio $\left(\mathrm{EO}_{2}\right)$ $\left(^{*}\right)$ diferença significativa $(p<0,05)$ com relação à inicial

Após a expansão do volume intravascular (M2) com solução fisiológica normal ou solução hipertônica de cloreto de sódio, quase todos os parâmetros voltaram aos níveis iniciais (Figuras 2 e 3 ). Após a reanimação a PAM estava discretamente reduzida em comparação com os valores iniciais nos dois grupos, mas essa diferença não foi estatisticamente significativa. Como pode ser visto nas figuras 2 e 3 , tanto a solução fisiológica normal quanto a solução hipertônica de cloreto de sódio restauraram a PAPM e a IRVP 
aos níveis iniciais, sendo esses valores um pouco maiores nos animais tratados com a solução hipertônica, mas não houve diferenças estatísticas significativas entre os grupos. A freqüência cardíaca permaneceu inalterada nos dois grupos. $\mathrm{O} \mathrm{VO}_{2}$ e $\circ \mathrm{DO}_{2}$ aumentaram enquanto $\circ \mathrm{EO}_{2}$ mostrou redução significativa após a administração das duas soluções, sem diferenças entre os dois grupos (Figura 4). A tabela II mostra que houve aumento significativo nos níveis plasmáticos de sódio no grupo tratado com $\mathrm{NaCl}$ a $7,5 \%$ em comparação com os valores obtidos antes de sua administração $(153,1 \pm 4,5 \mathrm{mEq} / \mathrm{L}$ vs. $142 \pm 3,8 \mathrm{mEq} / \mathrm{L}, \mathrm{p}<0,001) \mathrm{e}$ em comparação com o grupo tratado com $\mathrm{NaCl}$ a $0,9 \%$ $(153,1 \pm 4,5 \mathrm{mEq} / \mathrm{L}$ vs. $143 \pm 3 \mathrm{mEq} / \mathrm{L}, \mathrm{p}<0,001)$. Essa diferença permaneceu elevada até o final do experimento.

A PAM permaneceu inalterada em todos os grupos após a indução anestésica usando cetamina ou etomidato (Figura
2). A PVC aumentou no grupo tratado com $\mathrm{NaCl}$ a $7,5 \%$ e que recebeu cetamina em comparação com os outros grupos. As figuras 2 e 3 mostram que a PCP, PAPM e IRVP aumentaram de forma significativa nos animais anestesiados com cetamina, mas diminuíram nos animais tratados com etomidato 5 (M3) e 15 minutos (M4) após a administração do anestésico. A FC aumentou significativamente nos grupos tratados com cetamina, mas diminuíram após a administração de etomidato (Figura 3). O IC e IRVS (Figura 3) permaneceram estáveis em todos os grupos após a administração dos anestésicos. Após a reanimação volumétrica, com $\mathrm{NaCl}$ a $7,5 \%$ ou com $\mathrm{NaCl}$ a $0,9 \%$, os índices de transporte de oxigênio e de consumo de oxigênio, assim como a extração de oxigênio retornaram aos níveis iniciais, permanecendo inalterados, em M3 e M4, após a indução anestésica com os dois medicamentos (Figura 4).

Tabela II - Efeitos da Hipovolemia e da Administração Subseqüente de $\mathrm{NaCl}$ a $0,9 \%$ ou $\mathrm{NaCl}$ a $7,5 \%$ nas Concentrações Plasmáticas de Sódio e de Hemoglobina (Média \pm DP)

\begin{tabular}{lcccc}
\hline Variáveis Metabólicas & Inicial & $\begin{array}{c}\text { Imediatamente após } \\
\text { a Hemorragia }\end{array}$ & $\begin{array}{c}\text { Grupo Tratado } \\
\text { com NaCl a } 7,5 \%\end{array}$ & $\begin{array}{c}\text { Grupo Tratado } \\
\text { com NaCl a 0,9\% }\end{array}$ \\
\hline $\mathrm{Hb}\left(\right.$ g.dL $\left.{ }^{-1}\right)$ & $11,82 \pm 2,31$ & $13,49 \pm 2,81^{\mathrm{a}}$ & $9,72 \pm 1,84^{\mathrm{ab}}$ & $8,34 \pm 0,99^{\mathrm{a}}$ \\
$\mathrm{Na}^{+}\left(\mathrm{mEg} \cdot \mathrm{L}^{-1}\right)$ & $142,03 \pm 3,85$ & $142,34 \pm 3,76$ & $153,03 \pm 4,55^{\mathrm{ab}}$ & $143,13 \pm 3,3$ \\
\hline
\end{tabular}

$\mathrm{NaCl}$ a $0,9 \%$ : solução fisiológica; $\mathrm{NaCl}$ a $7,5 \%$ : solução hipertônica; $\mathrm{Hb}$ : concentração de hemoglobina; $\mathrm{Na}^{+}$: nível sérico de sódio. $a$ - diferença significativa $(p<0,05)$ em relação aos valores iniciais; $b$ - diferença significativa $(p<0.05)$ em relação ao grupo tratado com $\mathrm{NaCl}$ a $0,9 \%$.

\section{DISCUSSÃO}

Os principais resultados desse estudo foram: 1) tanto a solução fisiológica normal quanto a hipertônica de cloreto de sódio, em doses calculadas para fornecer a mesma quantidade de sal, reverteram o choque hemorrágico; 2) após o choque hemorrágico, os animais tratados com solução hipertônica de cloreto de sódio apresentaram um discreto aumento na PAPM e IRVP em comparação com o grupo tratado com solução fisiológica; 3) após a reanimação, nem a cetamina nem o etomidato interferiram de maneira significativa nos parâmetros hemodinâmicos e do transporte de oxigênio.

O objetivo deste estudo foi o de simular uma situação clínica encontrada com freqüência durante o tratamento de pacientes com trauma grave, quando grandes volumes de soluções venosas são administrados para o restabelecimento dos padrões hemodinâmicos e do transporte de oxigênio na emergência antes da indução anestésica para o tratamento cirúrgico. Os efeitos da interação entre o tipo de solução venosa utilizada e o anestésico nunca foram estudados. Em uma tentativa de evitar a confusão causada pelos efeitos hemodinâmicos da administração de tiopental e da ventilação mecânica necessária durante a cateterização vascular, o animal foi preparado com 24 horas de antecedência. No modelo experimental de choque hemorrágico utilizado nesse estudo ${ }^{11}$, o sangramento médio durante 0 estudo (30,4 mL. $\left.\mathrm{kg}^{-1}\right)$ e o subseqüente choque hipovolêmico com alterações hemodinâmicas foram comparáveis com os relatados na literatura ${ }^{8,9}$. Foi administrada pequena dose de morfina ao animal uma hora antes do experimento principal. O objetivo de sua administração foi o de manter os animais quietos durante o transporte para a sala do experimento, posicionamento na mesa e durante a fase de hemorragia enquanto permaneciam acordados. Pode-se fazer uma crítica com relação a esse estudo a respeito da influência da morfina nos parâmetros hemodinâmicos mas, de acordo com a literatura, as doses empregadas não parecem produzir qualquer efeito hemodinâmico significativo ${ }^{12}$. Como descrito na literatura'13,14, após a indução de um choque hemorrágico pressão-controlado ocorre redução significativa na PAM, IC, PCP, PAPM e no transporte e consumo de oxigênio, assim como um aumento na IRVS e IRVP. Como esperado, as duas soluções foram igualmente eficazes na reanimação do choque hemorrágico. A hemodiluição aguda com as duas soluções explicaria por que a PAM e o IRVS estavam menores do que os valores iniciais após a reanimação. Esses resultados foram semelhantes aos re- 
latados por diversos autores ${ }^{13-15}$. As duas soluções, em doses calculadas de forma a fornecer a mesma quantidade de sal, restauraram a PCP e a PVC aos valores iniciais, ao contrário do que foi previamente relatado por Prist e col., que encontraram maior aumento na PVC de animais reanimados com soluções hipertônicas de cloreto de sódio ${ }^{16}$. Um dos efeitos mais constantes das soluções hipertônicas nos modelos de choque hemorrágico é um aumento na pressão arterial e no débito cardíaco atribuído à mudança de líquido do compartimento extravascular para o intravascular ${ }^{5}$. Apesar de os dois grupos terem recebido a mesma quantidade de sal, foi infundido um volume de água maior no grupo que recebeu $\mathrm{NaCl}$ a $0,9 \%$. Isso pode explicar a diferença na concentração plasmática de sódio observada após a infusão de volume nos dois grupos, confirmando os resultados de outros estudos ${ }^{11,14}$. Houve redução significativa na resistência vascular periférica após a infusão das duas soluções. Sabe-se que a administração rápida de solução hipertônica produz uma vasodilatação acentuada conforme já foi relatado por Kien e col. ${ }^{17}$. O tempo prolongado de infusão deste estudo (10 minutos) pode explicar a ausência desse efeito nos animais. Por outro lado, a redução na IRVP após a administração de $\mathrm{NaCl}$ a $0,9 \%$ foi maior do que após a administração de $\mathrm{NaCl}$ a $7,5 \%$. Uma acentuação da vasoconstrição pulmonar secundária à hipóxia após a infusão de solução hipertônica de cloreto de sódio, como foi sugerido por Bellezza e col. ${ }^{18}$, explicaria a redução observada na IRVP. Esses autores descobriram que o uso da solução fisiológica hipertônica com dextran para reposição hídrica na hemodiluição isovolêmica aumentava a magnitude da vasoconstrição pulmonar secundária à hipóxia em leitões, enquanto a solução de dextran causava redução. Esse fato pode ser explicado pelos valores discretamente maiores da pressão arterial pulmonar média nos cães tratados com solução de $\mathrm{NaCl}$ a $7,5 \%$. Apesar dos efeitos da solução hipertônica na circulação pulmonar, as duas soluções restauraram efetivamente e de maneira semeIhante a distribuição e o consumo de oxigênio nos animais em choque.

Após a indução anestésica observou-se que a cetamina causou aumento significativo na PVC, PAP, IRVP, PCP e FC que poderia estar relacionada com a ativação simpática induzida por esse medicamento. Esses achados foram consistentes com os resultados obtidos por outros autores que usaram um modelo experimental semelhante ${ }^{19}$. Por outro lado, a administração de etomidato não causou alterações significativas nesses parâmetros hemodinâmicos ${ }^{9}$. Apesar dos distúrbios hemodinâmicos nas pressões de enchimento e na FC causados pela cetamina, a pressão arterial média, IC e IRVS permaneceram estáveis em todos os grupos após a indução anestésica. Foi descrito que a cetamina pode causar uma redução na PAM, diretamente proporcional à dose administrada, quando utilizada em animais com hipovolemia grave sem que seja feita a expansão volêmica prévia ${ }^{20}$, pois, apesar de sua ativação simpática, ela causa depressão do miocárdio. A administração de cetamina a suínos e cães submetidos ao choque hipovolêmico está associada a redução significativa da PAM após 5 minutos $^{8,21}$. Em cães hipovolêmicos, doses pequenas $\left(10 \mathrm{mg} \cdot \mathrm{kg}^{-1}\right)$ parecem estimular o sistema cardiovascular causando taquicardia, enquanto doses altas (20 mg. $\mathrm{kg}^{-1}$ ) podem causar depressão miocárdica profunda com bradicardia ${ }^{20}$. Nesse estudo, a cetamina só foi utilizada após a correção do choque hipovolêmico com soluções de cloreto de sódio isotônica ou hipertônica. A reposição adequada de fluidos, associada a um aumento na estimulação simpática induzida pela cetamina, explicaria a estabilidade da PAM e do IC. O efeito da cetamina no sistema nervoso simpático pode, em teoria, causar um aumento da IRVS 9 e a manutenção da PAM, mesmo na presença de um IC reduzido. O interessante é que a IRVS foi semelhante em todos os grupos. Isso se deve provavelmente à reposição adequada de fluidos que poderia ter contrabalançado os efeitos hemodinâmicos marginais dos agentes anestésicos.

Foi observado aumento significativo na PCP nos animais que receberam cetamina ao serem comparados com os grupos que receberam etomidato. Pode-se teorizar que o aumento da FC associado ao da contratilidade cardíaca induzido pelo aumento da atividade simpática causaria alguma disfunção diastólica, além de haver uma elevação da PAP mediada pelo sistema simpático causando um aumento da PCP. Houve aumento significativo da IRVP nos animais tratados com cetamina enquanto naqueles tratados com etomidato houve uma redução, que não foi estatisticamente significativa. Esses resultados são semelhantes aos obtidos em outras experiências usando cetamina para indução anestésica ${ }^{9,21-23}$. Nos animais tratados com solução hipertônica, a acentuação da vasoconstrição causada pela hipóxia poderia contribuir para o aumento da IRVP ${ }^{18}$. Infelizmente, não se pode confirmar a hipótese dos efeitos aditivos já que a IRVP não diferiu entre os animais que foram reanimados com solução isotônica ou hipertônica de cloreto de sódio. Por outro lado, a pequena redução do IRVP nos grupos que receberam etomidato poderia refletir a vasodilatação causada pela ausência de ativação do sistema nervoso simpático. Isso confirma os dados de alguns estudos experimentais nos quais os modelos de hipovolemia documentaram uma redução no IRVP após a infusão de etomidato. Os autores atribuíram a redução no IRVP à redução no tônus simpático induzida por esse agente ${ }^{9}$.

Concluiu-se que após o choque hipovolêmico os animais tratados com solução hipertônica de cloreto de sódio apresentaram um aumento discreto da PAP e IRVP em comparação com o grupo que recebeu solução fisiológica. Os grupos tratados com cetamina (solução fisiológica hipertônica) apresentaram aumento discreto, mas estatisticamente significativo, na PCP, PAPM, FC e IRVP sem efeitos clínicos relevantes. Nem a cetamina nem o etomidato apresentaram uma interferência significativa no transporte de oxigênio. Em virtude da diferença entre as espécies, é difícil transferir esse cenário 
para os pacientes que sofreram um trauma e que precisem de anestesia ou procedimentos cirúrgicos após a reanimação. Entretanto, pode ser importante levar em consideração a presença de alterações hemodinâmicas discretas observadas com os dois agentes nesse estudo ao tratar pacientes que precisem de cirurgia após situações que causaram choque hemorrágico grave, como trauma ou ferimentos por arma de fogo em civis ou militares.

\section{Anesthetic Induction after Treated He- morrhagic Shock: Experimental Study Comparing Ketamine and Etomidate}

Adilson O. Fraga, M.D.; Luiz Marcelo Sá Malbouisson, TSA, M.D.; Ricardo Prist, M.D.; Maurício Rocha e Silva, M.D.; José Otávio Costa Auler Júnior, TSA, M.D.

\section{INTRODUCTION}

Hemorrhagic shock due to trauma is an important cause of emergency hospital admission. Surgical intervention to control bleeding is often necessary, and anesthetic induction may cause further hemodynamic imbalance due to vasodilatation and myocardial depression. In such patients, vigorous crystalloid infusion using either isotonic or hypertonic crystalloid solutions has been used for initial resuscitation in emergency departments before the surgical intervention ${ }^{1,2}$. Hypertonic saline has been demonstrated in experimental and clinical studies to be efficient to restore hemodynamic stability after hemorrhagic shock ${ }^{3-5}$, but isotonic saline continues to be the main fluid used during initial resuscitation of hemorrhagic shock ${ }^{1}$. Controversy also remains regarding the optimal choice of an anesthetic induction drug that will minimally interfere with the hemodynamic state mainly in traumatized hypovolemic patients ${ }^{6,7}$. In 1990, Haskins et al. studied the effects of ketamine in a model of hypovolemic dogs and observed that it adequately maintained cardiovascular function, preserved oxygen transport, and causes only transient respiratory depression ${ }^{8}$. Based on these data, ketamine has been considered one option for the induction of anesthesia in hypovolemic patients in emergency situations. Etomidate, another anesthetic agent, has been considered safe for use in critically ill patients, because autonomic nervous system reflexes are preserved as well as myocardial contractility $^{6}$. Despite the widespread use of both anesthetic agents in cases of unstable circulation, few studies have compared these drugs in experimental models of hemorrhagic shock and their interactions with normal saline and hypertonic sodium chloride used to treat hemorrhagic shock ${ }^{8-10}$. The purpose of this study was to compare the hemodynamic effects of ketamine and etomidate in dogs submitted to a model of hemorrhagic shock, after being resuscitated with normal saline or hypertonic sodium chloride $(\mathrm{NaCl} 7.5 \%)$ solutions.

\section{METHODS}

This study was approved by the Hospital Ethics Committee and was in agreement with the international rules for animal experimental research. The study was performed in 32 male mongrel dogs, weighing between 15 and $20 \mathrm{~kg}$. Dogs were fasted for 12 hours before the study, with free access to water. To avoid possible influence with different anesthetics the animals were prepared under sedation with thiopental (15 mg. $\left.\mathrm{kg}^{-1}\right)$, and mechanical ventilation twenty-four hours before the experiment. A 7-French pulmonary artery catheter (Baxter Healthcare Corp., Irvine, CA, USA) was inserted via the right internal jugular vein and positioned in the pulmonary artery under fluoroscopic visualization. Small gauge polyethylene catheters (P260) filled with $10 \mathrm{UI} . \mathrm{mL}^{-1}$ heparin were inserted, by dissection, into both femoral arteries and veins. The arterial catheters were used to measure the systemic arterial pressure, remove blood, and collect arterial blood sample as well as for drug administration and volume expansion. All catheters were firmly secured and tunneled subcutaneously and exteriorized in the dorsal region of each animal for later use. After all vascular accesses were obtained, $50 \mathrm{Ul}^{\mathrm{kg}} \mathrm{kg}^{-1}$ heparin was administered via the central venous route and the animals were subsequently extubated and allowed to recover during 24 hours with free access to water. After this period of resting, the dogs were pre-medicated with morphine $\left(0.4 \mathrm{mg} \cdot \mathrm{kg}^{-1}\right)$ one hour before the transportation to experimentation room, where the main study was carried out. Arterial and mixed venous oxygen $\left(\mathrm{PaO}_{2}, \mathrm{PvO}_{2}\right)$, carbon dioxide $\left(\mathrm{PaCO}_{2}, \mathrm{PvCO}_{2}\right)$ tensions and plasmatic sodium $\left(\mathrm{Na}^{+}\right)$level, as well as the arterial and mixed venous $\mathrm{pH}$ values, were measured using a blood-gas analyzer (Gas Analyzer ABL Radiometer, Copenhagen). All measurements were corrected for body temperature, and the sodium bicarbonate concentration and base excess were calculated for each animal. Hemoglobin concentrations $(\mathrm{Hb})$ were also determined. The mean arterial pressure (MAP), mean pulmonary artery pressure (MPAP), pulmonary capillary wedge pressure (PCWP), and central venous pressure (CVP) were measured using pressure transducers, by means of the Acknowledge MP 100 (Biopac Systems Inc, Goleta, CA). Cardiac output (CO) was measured using the standard thermodilution technique. The measure was repeated five times, and the average value was divided by body surface area to obtain the cardiac index $(\mathrm{Cl})$. Systemic and pulmonary vascular resistances, arterial and mixed venous blood oxygen contents $\left(\mathrm{CaO}_{2}\right.$ and $\left.\mathrm{CvO}_{2}\right)$, the venous admixture, oxygen consumption $\left(\mathrm{VO}_{2}\right)$, oxygen delivery, and oxygen utilization ratio were all calculated from measured values utilizing conventional equation.

The model of hemorrhagic shock used was the one described by Prist et al. ${ }^{11}$. In this model, the animal was submitted to continuous bleeding throughout the procedure, and a small blood sample was removed each minute. The prevailing pressure during the last 30 seconds (30s) of the minute prior to each bleeding event was used to establish the blood vo- 
lume to be removed during the first 30 s of the next minute. Thus, the volume of the blood sample corresponds to the previous MAP. The described model is derived from a standard situation in which the removal of an initial blood volume of $25 \mathrm{~mL} . \mathrm{min}^{-1}$ from $17.5 \mathrm{~kg}$ dogs with a MAP of 100 $\mathrm{mmHg}$ corresponds to a blood loss of $100 \mathrm{~mL}^{\mathrm{min}} \mathrm{m}^{-1}$ for a 70 $\mathrm{kg}$ adult with the same MAP. The different animal weights or MAP variations were taken into consideration for determining the rate of bleeding according to the following conditions:

1. The MAP was determined during the 5 minutes preceding the beginning of hemorrhage;

2. An initial bleeding volume ( $\mathrm{VbO}$ ) was adjusted to the actual body weight;

Since the target of shock is the achievement of a mean arterial pressure of $40 \mathrm{mmHg}$ during bleeding, individual variation in total shed blood volume is expected in order to maintain the same MAP.

The animals were studied in relation to diverse hemodynamic variables assessed at five experimental times, considered from M0 - baseline to M4:

(M0) Baseline;

(M1) After bleeding (hemorrhagic shock);

(M2) After volume expansion (normal and hypertonic sodium chloride solutions);

(M3) 5 minutes after infusion of anesthetic agent (etomidate or ketamine) and tracheal intubation;

(M4) 15 minutes after infusion of anesthetic agent;

The experimental procedure is shown in the Figure 1. After obtaining the baseline hemodynamic measurements (M0), all animals were submitted to hemorrhagic shock as des- cribed in hemorrhage protocol section. After achieving the target mean arterial pressure of $40 \mathrm{mmHg}, 30$ minutes after the beginning of the experiment (M1), new hemodynamic measurements were obtained and blood samples were collected. At this moment, the animals were divided in two groups according to expansion solution they were assigned to receive: 16 dogs received $\mathrm{NaCl} 0.9 \%\left(32 \mathrm{~mL} . \mathrm{kg}^{-1}\right)$ and 16 received $\mathrm{NaCl} 7.5 \%\left(4 \mathrm{~mL}^{\mathrm{kg}}{ }^{-1}\right)$ during ten minutes. The volumes of normal and hypertonic saline solution were chosen in order to give the same $\mathrm{NaCl}$ load to both groups. After volemic expansion (M2), other hemodynamic measurements were obtained and arterial and venous blood samples collected. In the next step, each group was further split in two subgroups assigned to receive either etomidate $\left(1 \mathrm{mg} \cdot \mathrm{kg}^{-1}\right.$, $\mathrm{N}=8$ ) or ketamine (4 mg. $\mathrm{kg}^{-1}, \mathrm{n}=8$ ) as anesthetic induction agent, followed by tracheal intubation. M3 and M4 measures were collected 5 and 15 minutes after anesthetic injection respectively. After $\mathrm{M} 2$, the four groups of animals were labeled as $\mathrm{GI}$ ) $\mathrm{NaCl} 0.9 \%$ and ketamine; GII) $\mathrm{NaCl} 7.5 \%$ and ketamine; GIII) $\mathrm{NaCl} 0.9 \%$ and etomidate; and GIV) $\mathrm{NaCl}$ $7.5 \%$ and etomidate.

Statistical analyses were performed using the SPSS 10 statistical package (SPSS Inc., Cary, Ca). One-way analysis of variance (ANOVA) was used to compare weight, body surface area and total blood loss among the four groups. Two-way ANOVA for repeated measures followed by Student-Newmann post-hoc test when indicated was used to analyze the hemodynamic parameters. A $p$ value of $<0.05$ was considered statistically significant. All data are presented as mean $\pm \mathrm{SD}$.

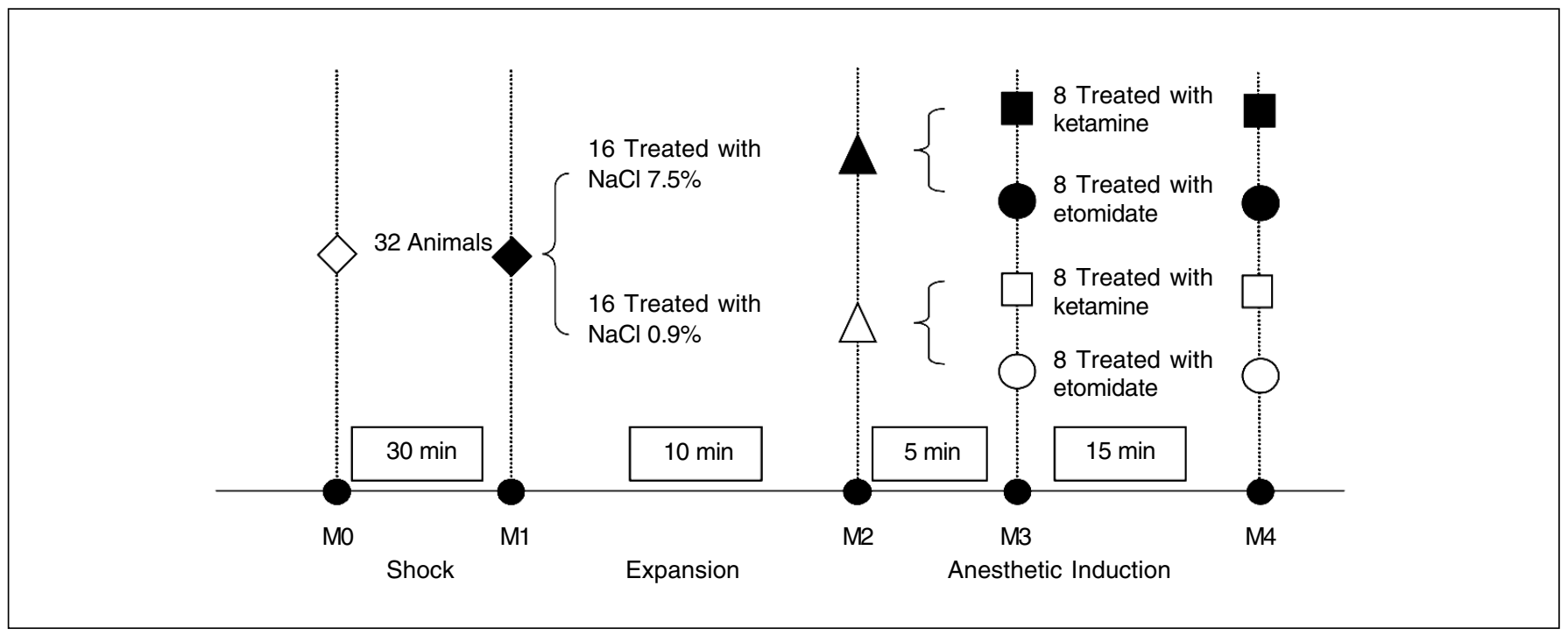

Figure 1 - Experimental Model.

M0: baseline; M1: 30 min after bleeding; M2: 10 min after volume expansion; M3: 5 min after anesthetic induction and tracheal intubation; M4: 15 min after anesthetic induction and tracheal intubation. Open diamonds (all animals - baseline), Closed diamonds (all animals - after shock), Open triangles ( $\mathrm{NaCl} 0.9 \%$ treated animals), closed triangles $(\mathrm{NaCl} 7.5 \%$ treated animals) open squares $(\mathrm{G} \mathrm{I}-\mathrm{NaCl} 0.9 \% / \mathrm{ketamine}$ treated animals), closed squares (G II - NaCl $7.5 \%$ / ketamine treated animals), open circles (G III - NaCl $0.9 \%$ / etomidate treated animals), and closed circles (G IV - NaCl $7.5 \%$ / etomidate treated animals). 
During baseline (M0) and M1, all animals were submitted to the same experimental procedures and data collection. For statistical analysis they were considered as a single group. During M2, for the statistical analysis, the animals were divided in two groups according to the fluid infusion: 16 animals received $\mathrm{NaCl} 0.9 \%$ and 16 animals received $\mathrm{NaCl} 7.5 \%$. After M2, each group of 16 animals was divided again in two subgroups $(n=8)$ according to the anesthetic drug, etomidate or ketamine. During M3 and M4, the groups, as previously defined $\mathrm{Gl}$ ) $\mathrm{NaCl} 0.9 \%$ and ketamine; GII) $\mathrm{NaCl} 7.5 \%$ and ketamine; GIII) $\mathrm{NaCl} 0.9 \%$ and etomidate; and GIV) $\mathrm{NaCl}$ $7.5 \%$ and etomidate were submitted to statistical analysis.

\section{RESULTS}

Table I summarizes characteristics of the animals, including body weight, body surface area, and volume of blood removed. Values for body surface area and shed blood volume did not significantly differ between groups.

As shown in the Figures 2, 3 and 4, hemodynamic signals of severe blood loss were observed. Compared to baseline (M0), significant decreases in CVP, MAP, Cl, PCWP and MPAP $(p<0.001)$ and significant increases in PVRI and SVRI $(p<$ 0.001 ) were observed, while HR remained unaltered. Parameters, representing oxygen delivery $\left(\mathrm{DO}_{2}\right)$, and oxygen consumption $\left(\mathrm{VO}_{2}\right)$ decreased significantly $(\mathrm{p}<0.001)$, while a significant increase in oxygen extraction $\left(\mathrm{OERO}_{2}\right)(p<0.001)$ was observed.

Figure 2 - Mean Arterial Pressure (MAP), Mean Pulmonary Artery Pressure (MPAP), Central Venous Pressure (CVP) and Pulmonary Capillary Wedge Pressure (PCWP).

Treatment given at M2. Open diamonds (all animals - baseline), closed diamonds (all animals - after shock), open triangles ( $\mathrm{NaCl}$ $0.9 \%$ treated animals), closed triangles ( $\mathrm{NaCl} 7.5 \%$ treated animals) open squares ( $\mathrm{Gl}-\mathrm{NaCl} 0.9 \%$ / ketamine treated animals), closed squares (GII - NaCl 7.5\% / ketamine treated animals), open circles (GIII - NaCl 0.9\% / etomidate treated animals), and closed circles (G IV - $\mathrm{NaCl} 7.5 \%$ / etomidate treated animals).

$\left.{ }^{*}\right)$ significantly different $(p<0.05)$ from baseline.

$(+)$ significantly different between ketamine and etomidate treated groups $(p<0.05)$.

$(\Delta)$ significant differences between GII and other groups after anesthetic induction $(p<0.05)$.



Table I - Animals Characteristics (Mean \pm SD)

\begin{tabular}{lcccc}
\hline & Group I $(\mathrm{n}=8)$ & Group II $(\mathrm{n}=8)$ & Group III $(\mathrm{n}=8)$ & Group IV $(\mathrm{n}=8)$ \\
\hline Weight $(\mathrm{kg})$ & $17.36 \pm 0.37$ & $17.46 \pm 0.50$ & $17.31 \pm 0.49$ & $17.79 \pm 0.54$ \\
Body surface areas $\left(\mathrm{kg} \cdot \mathrm{m}^{-2}\right)$ & $0.82 \pm 0.01$ & $0.82 \pm 0.16$ & $0.82 \pm 0.15$ & $0.83 \pm 0.17$ \\
Total volume of blood loss $\left(\mathrm{mL} \cdot \mathrm{kg}^{-1}\right)$ & $28.84 \pm 1.47$ & $28.92 \pm 1.75$ & $30.44 \pm 2.0$ & $30.01 \pm 1.81$ \\
\hline
\end{tabular}

Group I: normal saline ( $\mathrm{NaCl} 0.9 \%)$ and ketamine; Group II: hypertonic saline ( $\mathrm{NaCl} 7.5 \%)$ and ketamine; Group III: $\mathrm{NaCl} 0.9 \%$ and etomidate; Group IV: $\mathrm{NaCl} 7.5 \%$ and etomidate 


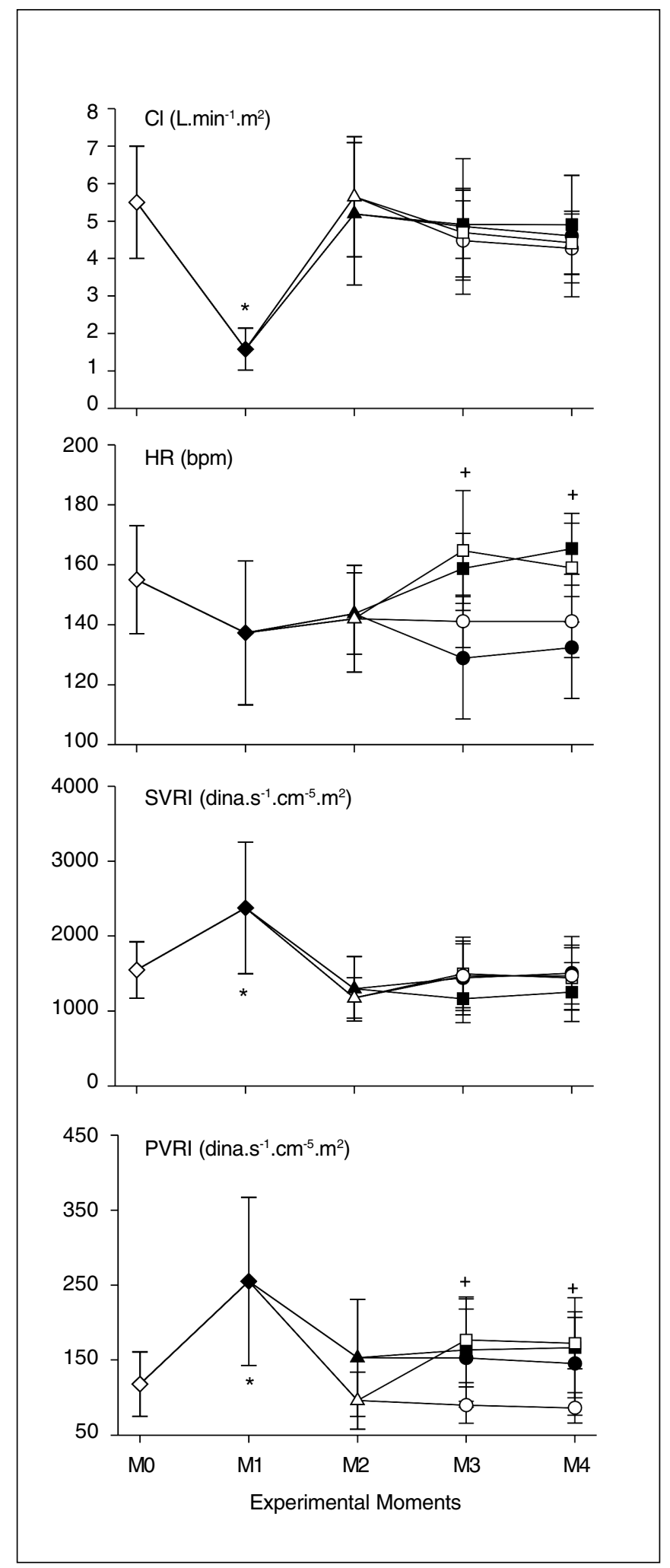

Figure 3 - Cardiac Index (Cl), Heart Rate (HR), Systemic Vascular Resistance Index (SVRI) and Pulmonary Vascular Resistance Index (PVRI)

$\left.{ }^{*}\right)$ significantly different $(p<0.05)$ from baseline.

$(+)$ significantly different between ketamine and etomidate treated groups $(p<0.05)$.

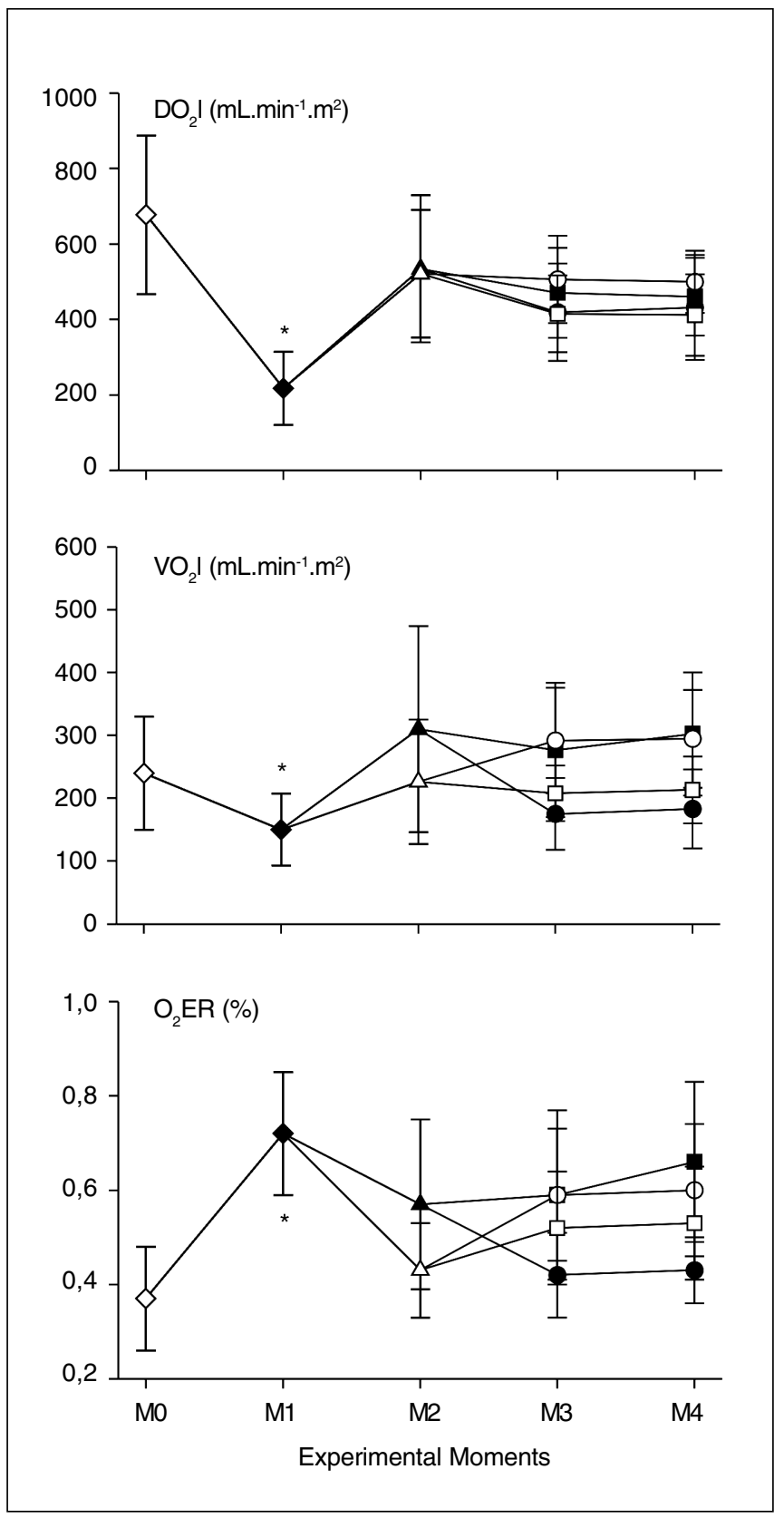

Figure 4 - Oxygen Transport Index $\left(\mathrm{DO}_{2}\right)$, Oxygen Consumption index $\left(\mathrm{VO}_{2}\right)$ Oxygen Extraction $\left(\mathrm{O}_{2} \mathrm{ER}\right)$

$\left(^{*}\right)$ significantly different $(p<0.05)$ from baseline.

After intravascular expansion (M2) either with normal saline or hypertonic sodium chloride, almost all parameters were restored to baseline (Figures 2 and 3). After resuscitation, MAP was slightly lower when compared to baseline measurement in both groups, although without statistical significance. As shown in figures 2 and 3, both normal and hypertonic saline infusions restored MPAP and PVRI compared to initial values, being slightly greater in hypertonic treated animals, but without significant differences between groups. Heart 
rate remained unaltered in both groups. $\mathrm{VO}_{2}, \mathrm{DO}_{2}$ increased and $\mathrm{O}_{2} \mathrm{ER}$ decreased significantly following the administration of both solutions without difference between groups (Figure 4). As shown in Table II, there was a significant increase in plasma sodium levels in the group treated with $\mathrm{NaCl} 7.5 \%$ solution when compared to pre-infusion values $(153.1 \pm 4.5 \mathrm{mEq} / \mathrm{L}$ vs. $142 \pm 3.8 \mathrm{mEq} / \mathrm{L}, \mathrm{p}<0.001)$ and when compared to the $\mathrm{NaCl} 0.9 \%$ solution treated group (153.1 \pm $4.5 \mathrm{mEq} / \mathrm{L}$ vs. $143.1 \pm 3 \mathrm{mEq} / \mathrm{L}, \mathrm{p}<0.001)$. This difference remained elevated until the end of experimental protocol.

MAP remained unaltered in all groups following anesthetic induction using either ketamine or etomidate (Figure 2). CVP increased in the $\mathrm{NaCl} 7.5 \%$ treated group branch that received ketamine when compared to others. As shown in figure 2 and 3, PCWP, MPAP, and PVRI significantly increased in the animals anesthetized with ketamine, but decreased in the animals treated with etomidate at five (M3) and 15 minutes (M4) after anesthetic injection. HR increased significantly in the ketamine treated groups, but decreased following etomidate infusion (Figure 3). Cl and SVRI (Figure 3) remained stable in all groups after anesthetic administration. After fluid resuscitation, either with $\mathrm{NaCl} 7.5 \%$ or $\mathrm{NaCl}$ $0.9 \%$, oxygen transport index, oxygen consumption index and oxygen extraction were restored to baseline levels and remained unaltered after anesthetic induction with both drugs in M3 and M4 (Figure 4).

Table II - Effect of Hypovolemia and the Subsequent Administration of $\mathrm{NaCl} 0.9 \%$ or $\mathrm{NaCl} 7.5 \%$ on Plasma Sodium and Hemoglobin Concentrations (Mean \pm SD)

\begin{tabular}{lcccc}
\hline Metabolic Variables & Baseline & $\begin{array}{c}\text { Immediately } \\
\text { Posthemorrhage }\end{array}$ & $\begin{array}{c}\text { NaCl } 7.5 \% \\
\text { Treated Group }\end{array}$ & $\begin{array}{c}\text { NaCl 0.9\% } \\
\text { Treated Group }\end{array}$ \\
\hline $\mathrm{Hb}\left(\mathrm{g} \cdot \mathrm{dL}^{-1}\right)$ & $11.82 \pm 2.31$ & $13.49 \pm 2.81^{\mathrm{a}}$ & $9.72 \pm 1.84^{\text {a b }}$ & $8.34 \pm 0.99 \mathrm{a}$ \\
$\mathrm{Na}^{+}\left(\mathrm{mEq} \cdot \mathrm{L}^{-1}\right)$ & $142.03 \pm 3.85$ & $142.34 \pm 3.76$ & $153.03 \pm 4.55^{\text {a b }}$ & $143.13 \pm 3.3$ \\
\hline
\end{tabular}

$\mathrm{NaCl}$ 0.9\%: normal saline; $\mathrm{NaCl} 7.5 \%$ : hypertonic saline; $\mathrm{Hb}$ : hemoglobin concentration; $\mathrm{Na}^{+}$: serum sodium level. a - significantly $(\mathrm{p}<0.05)$ different from baseline value; $b$ - significantly $(p<0.05)$ different from $\mathrm{NaCl} 0.9 \%$ :treated group.

\section{DISCUSSION}

The main results of this study were: 1) either normal saline or hypertonic saline solutions in doses calculated to offer the same load of salt reversed hemorrhagic shock 2) after hemorrhagic shock animals treated with hypertonic saline solution presented a slight increase in the values of MPAP and PVRI when compared to the normal saline group 3) after resuscitation, neither ketamine nor etomidate significantly interfered with hemodynamic parameters and oxygen transportation.

In this study, we aimed to simulate a clinical situation that is commonly encountered during the treatment of patients with severe trauma, when large volumes of crystalloid are given to reestablish hemodynamics and oxygen transport in the emergency room before anesthetic induction for surgical treatment. The effects of interaction between the kind of volume expansion solution and the anesthetic induction drug have never been studied before. As an attempt to avoid the confounding effects on hemodynamics of thiopental infusion and mechanical ventilation necessary during vascular catheterization, the animal preparation took place 24 hours prior to the experiment. In the experimental model of hemorrhagic shock used in this study ${ }^{11}$, the mean bleeding during the study (30.4 mL. $\left.\mathrm{kg}^{-1}\right)$ and subsequent state of hypovolemic shock with hemodynamic alterations were comparable to those reported in the literature ${ }^{8,9}$. A small dose of morphine as premedication was administered to the animal's one hour before the main experiment. The aim of morphine admi- nistration was to keep animals quiet during transport to the experimentation room, positioning in the experiment table and during the hemorrhage phase, when they were still awake. One criticism to our study could be raised about the influence of morphine on the hemodynamic parameters, but according to the literature the doses employed do not seem to produce any significant hemodynamic effect ${ }^{12}$.

As previously described in the literature ${ }^{13,14}$, after the pressurecontrolled hemorrhagic shock induction, a significant decrease in the MAP, CI, PCWP, MPAP and in oxygen transport and consumption, as well as an increase in the SVRI and PVRI were observed. As expected, both solutions were equally effective in the resuscitation of hemorrhagic shock. Acute hemodilution with both solutions would explain how MAP and SVRI tended to be lower than baseline after resuscitation. These results were similar to those reported by several authors $^{13-15}$. Both solutions in doses calculated to offer the same load of salt equally restored PCWP and CVP baseline levels, in contrast to the data previously reported by Prist et al, that found a greater increase in CVP of animals resuscitated with hypertonic saline solution ${ }^{16}$. One of the most consistent effects of hypertonic solutions in hemorrhagic shock models has been an increase in the arterial pressure and cardiac output attributed to fluid shift from extravascular to intravascular compartment ${ }^{5}$. Although the same load of salt was given to both groups, a greater volume of water was infused in the $\mathrm{NaCl} 0.9 \%$ group. This may explain the difference in plasma sodium concentration observed after fluid infusion in both groups and is in concordance with 
results from other studies ${ }^{11,14}$. Systemic vascular resistance significantly decreased very similarly following the infusion of both solutions. Rapid infusion of hypertonic solution is known to induce profound vasodilatation, as previously reported by Kien et al. ${ }^{17}$. The prolonged infusion time used in our study (10 minutes) may explain the absence of this effect in our animals. On the other hand, the PVRI decrease after $\mathrm{NaCl}$ $0.9 \%$ solution infusion was greater then after $\mathrm{NaCl} 7.5 \%$. A reinforcement of hypoxic pulmonary vasoconstriction after hypertonic saline solution infusion, as suggested by Bellezza et al. $^{18}$, would explain the observed increase in PVRI. These authors found that hypertonic saline dextran solution, as replacement fluid in isovolemic hemodilution, increased the magnitude of hypoxic pulmonary vasoconstriction in piglets, whereas a dextran solution reduced it. This fact may be explained by the slightly higher values of mean pulmonary arterial pressure in dogs treated with $\mathrm{NaCl} 7.5 \%$ solution. Despite the effects of hypertonic solution on pulmonary circulation, both solutions effectively and similarly restored oxygen delivery and consumption in shocked animals.

After anesthetic induction, we observed that ketamine promoted a significant increase in CVP, PAP, PVRI, PCWP and $\mathrm{HR}$, which could be related to the ketamine-induced sympathetic activation. These findings were consistent with the results of other authors using similar experimental model ${ }^{19}$. On the other hand, etomidate infusion did not result in significant alterations in such hemodynamic parameters ${ }^{9}$. Despite the hemodynamic derangements in filling pressures and HR with ketamine, mean arterial pressure, $\mathrm{Cl}$ and SVRI remained stable in all groups after anesthesia induction. It has been described that ketamine may cause a dose-dependent decrease in MAP when administered to severely hypovolemic animals without prior volume expansion ${ }^{20}$, due to as myocardial depressor effect, despite the ketamine-induced sympathetic activation. The administration of ketamine to swine and dogs submitted to hypovolemic shock was associated with a significant fall in the MAP, 5 minutes after drug administration $^{8,21}$. In hypovolemic dogs, low doses (10 mg. $\mathrm{kg}^{-1}$ ) seem to stimulate the cardiovascular system, leading to tachycardia, While high doses of ketamine $\left(20 \mathrm{mg} \cdot \mathrm{kg}^{-1}\right)$ may cause profound myocardial depression with bradycardia $^{20}$. In this study, ketamine was only injected after correction of the hypovolemic shock with normal or hypertonic saline solutions. The adequate fluid replacement along with the increase in sympathetic tonus induced by ketamine would explain the stability of MAP and $\mathrm{Cl}$. The effect of ketamine on the sympathetic system might theoretically induce an increase on $\mathrm{SVRI}^{9}$, and the maintenance of MAP, even in the presence of a low $\mathrm{Cl}$. Interestingly, SVRI was comparable in all groups. This is probably due to the adequate fluid reposition, which might have counterbalanced marginal hemodynamic effects of the anesthetic agents.

A significant increase in the PCWP was observed in animals that received ketamine, when compared to the groups that received etomidate. We could hypothesize that the increase in HR associated to sympathetic-induced increase in contractility would promote some degree of diastolic dysfunction along with the sympathetic-mediated elevation of PAP would increase PCWP. The PVRI significantly increased in animals treated with ketamine while animals receiving etomidate experienced a less-than-statistically significant decrease in the PVRI. These results are in agreement with other experiments using ketamine as anesthetic induction agent ${ }^{9,21-23}$. In hypertonic solution treated animals, reinforcement of hypoxic pulmonary vasoconstriction could contribute to the increase of $P V R I^{18}$. Unfortunately, we cannot confirm the hypothesis of additive effects, since PVRI was not different in ketamine anesthetized animals that were resuscitated either with normal or hypertonic saline solution. On the other hand, the nonsignificant decrease in PVRI in the etomidate groups could reflect vasodilatation due to an absence of sympathetic activation. This is accordance with data from some experimental studies in hypovolemic state models that documented a decrease in PVRI after etomidate infusion. The authors attributed the decrease in PVRI to a reduction in sympathetic tone induced by this agent ${ }^{9}$.

In conclusion, after hemorrhagic shock, animals treated with hypertonic saline presented a slight increase in the values of PAP and PVRI when compared to normal saline group. Ketamine treated groups (saline and hypertonic) presented a discrete but statistically significant increase in PCWP, MPAP, $\mathrm{HR}$ and PVRI without clinical relevant effects. Neither ketamine nor etomidate significantly interfered with the oxygen transport status. Due to the difference of species it is difficult to transfer this scenario to the human traumatized patient who may require anesthesia or surgical procedures after rescue and reanimation. However, the findings of minor hemodynamic derangement observed with both anesthetic agents in our experimental study may be worthy of consideration when patients are required to have surgery after adverse situations that cause severe hemorrhagic shock such as trauma of civilian or military casualties caused by firearms.

\section{REFERÊNCIAS - REFERENCES}

01. Krausz MM - Controversies in shock research: hypertonic resuscitation - pros and cons. Shock, 1995;3:69-72.

02. Moss GS, Gould AS - Plasma expanders: an update. Am J Surg, 1988;155:425-434.

03. Krausz MM, Bashenko Y, Hirsh M - Crystalloid and colloid resuscitation of uncontrolled hemorrhagic shock following massive splenic injury. Shock, 2001;16:383-388.

04. Shi HP, Deitch EA, Da Xu Z et al - Hypertonic saline improves intestinal mucosa barrier function and lung injury after traumahemorrhagic shock. Shock, 2002;17:496-501.

05. de Felippe J Jr, Timoner J, Velasco IT et al - Treatment of refractory hypovolemic shock by $7.5 \%$ sodium chloride injections. Lancet, 1980;2:1002-1004.

06. Paes da Silva F, Gonzalez AP, Tibirica E - Effects of fluid resuscitation on mesenteric microvascular blood flow and lymphatic activity after severe hemorrhagic shock in rats. Shock, 19: $55-60$. 
07. Plewa MC, King R, Johnson D et al - Etomidate use during emergency intubation of trauma patients. Am J Emerg Med, 1997;15:98-100.

08. Haskins SC, Patz JD - Ketamine in hypovolemic dogs. Crit Care Med, 1990;18:625-629.

09. Pascoe PJ, Ilkiw JE, Haskins SC et al - Cardiopulmonary effects of etomidate in hypovolemic dogs. Am J Vet Res, 1992;53:21782182.

10. Gelissen HP, Epema AH, Henning RH et al - Inotropic effects of propofol, thiopental, midazolam, etomidate, and ketamine on isolated human atrial muscle. Anesthesiology, 1996; 84:397-403.

11. Prist R, Rocha e Silva M, Velasco IT et al - Pressure-driven hemorrhage: a new experimental design for the study of crystalloid and small-volume hypertonic resuscitation in anesthetized dogs. Circ Shock, 1992;36:13-20.

12. Priano LL, Vatner SF - Morphine effects on cardiac output and regional blood flow distribution in conscious dogs. Anesthesiology, 1981;55:236-243.

13. Velasco IT, Pontieri V, Rocha e Silva M Jr et al - Hyperosmotic $\mathrm{NaCl}$ and severe hemorrhagic shock. Am J Physiol, 1980; 239: H664-H673.

14. Rocha e Silva M, Braga GA, Prist R et al - Physical and physiological characteristics of pressure-driven hemorrhage. Am J Physiol, 1992;263:1402-1410.

15. Nakayama S, Sibley L, Gunther RA et al - Small volume resuscitation with hypertonic saline $(2400 \mathrm{mOsm} / \mathrm{liter})$ during hemorrhagic shock. Circ Shock, 1984;13:149-159.

16. Prist $R$, Rocha e Silva $M$, Scalabrini $A$ et al - $A$ quantitative analysis of transcapillary refill in severe hemorrhagic hypotension in dogs. Shock, 1994;1:188-195.

17. Kien ND, Kramer GC, White DA - Acute hypotension caused by rapid hypertonic saline infusion in anesthetized dogs. Anesth Analg, 1991;73:597-602

18. Bellezza M, Kerbaul F, Roussel $L$ et al - The effect of hypertonic saline dextran solutions on hypoxic pulmonary vasoconstriction in anesthetized piglets. Eur Respir J, 2002;20:965-971.

19. Tweed WA, Minuck M, Mymin D - Circulatory responses to ketamine anesthesia. Anesthesiology, 1972;37:613-619.

20. Chen TS, Yeh FC, Chou YP et al - Effects of ketamine on the circulatory functions and body tissue oxygenation in dogs under normal and hypovolemic conditions. Proc Natl Sci Counc Repub China B, 1984;8:168-176.

21. Weiskopf RB, Bogetz MS, Roizen MF et al - Cardiovascular and metabolic sequela of inducing anesthesia with ketamine or thiopental in hypovolemic swine. Anesthesiology, 1984;60:214-219.

22. Debaene B, Goldfarb G, Braillon A et al - Effects of ketamine, halothane, enflurane, and isoflurane on systemic and splanchnic hemodynamics in normovolemic and hypovolemic cirrhotic rats. Anesthesiology, 1990;73:118-124.

23. Criado A, Maseda J, Navarro E et al - Induction of anaesthesia with etomidate: haemodynamic study of 36 patients. $\mathrm{Br} \mathrm{J}$ Anaesth, 1980;52:803-806.

\section{RESUMEN}

Fraga AO, Malbouisson LMS, Prist R, Silva MR, Auler Jr JOC Inducción Anestésica después del Tratamiento del Choque Hemorrágico: Estudio Experimental Comparando la Cetamina y el Etomidato.

JUSTIFICATIVA Y OBJETIVOS: El sangramiento que conlleva al choque hemorrágico generalmente necesita un tratamiento quirúrgico bajo anestesia general. A su vez, los anestésicos pueden comprometer más las condiciones hemodinámicas. El objetivo de este estudio fue el de comparar los efectos hemodinámicos de la cetamina y del etomidato durante la inducción anestésica en perros sometidos a un modelo experimental de choque hemorrágico y reanimación.

MÉTODO: Treinta y dos perros mestizos fueron sometidos al choque hemorrágico presión-controlada, reanimación e inducción anestésica. Después de alcanzar la presión objeto de $40 \mathrm{mmHg}$ ellos fueron divididos aleatoriamente en dos grupos de acuerdo con el líquido usado en la resucitación: $\mathrm{NaCl}$ a $0.9 \%\left(32 \mathrm{~mL} . \mathrm{kg}^{-1}\right)$ y $\mathrm{NaCl}$ a 7,5\% (4 mL. $\left.\mathrm{kg}^{-1}\right)$. Después de la infusión de volumen, esos grupos fueron divididos nuevamente de acuerdo con el anestésico utilizado: GI) NaCl a $0.9 \%$ y cetamina; GII) NaCl a $7.5 \%$ y cetamina; GIII) $\mathrm{NaCl}$ a $0.9 \%$ y etomidato; $y$ GIV) $\mathrm{NaCl}$ a $7.5 \%$ y etomidato. Mediciones hemodinámicas fueron obtenidas en cinco momentos: (M0) inicial; (M1) después del desarrollo del choque hemorrágico; (M2) después de la administración de soluciones; (M3) 5 minutos después de la inducción anestésica; (M4) 15 minutos después de la inducción anestésica. Se hizo el análisis estadístico usando el Student t test y two-way ANOVA. Fueron considerados significativos valores de $p$ menores que 0,05.

RESULTADOS: Después de la instalación del choque, los dos sueros reestablecieron los estándares hemodinámicos a los valores iniciales. Independiente del anestésico o del solución utilizada, después de la inducción anestésica la presión arterial media permaneció inalterada en todos los grupos. La presión venosa central, frecuencia cardiaca, presión capilar pulmonar y el índice de resistencia pulmonar vascular aumentaron significativamente después de la administración de cetamina. El índice cardíaco, el índice de resistencia vascular sistémica y el transporte de oxígeno permanecieron estables en todos los grupos.

CONCLUSIONES: El etomidato o la cetamina fueron capaces de mantener la estabilidad hemodinámica en los perros que sufrieron choque hemorrágico severo y que fueron tratados con $\mathrm{NaCl}$ a $0,9 \%$ o $\mathrm{NaCl}$ a $7,5 \%$. 